\title{
Driver intoxication and risk for fatal crashes in South Africa: A 3-year review
}

\author{
R Govender, ${ }^{1,2} \mathrm{PhD}$; A Sukhai, ${ }^{1,2} \mathrm{PhD}$; D Roux, ${ }^{3}$ BSc Hons; A van Niekerk, ${ }^{1,2} \mathrm{PhD}$ \\ ${ }^{1}$ South African Medical Research Council-University of South Africa Violence, Injury and Peace Research Unit, Cape Town, South Africa \\ ${ }^{2}$ Institute for Social and Health Sciences, University of South Africa, Johannesburg, South Africa \\ ${ }^{3}$ Road Traffic Management Corporation, Pretoria, South Africa
}

Corresponding author: R Govender (govender@iafrica.com)

\begin{abstract}
Background. Globally, alcohol intoxication has been shown to be significantly associated with increased risk for road traffic crash morbidity and mortality for all road users (drivers, passengers and pedestrians). This association relates to the diminished capacity of drivers while intoxicated to operate motor vehicles and the increased propensity for risk-taking behaviours. The overall prevalence of alcohol-related fatal crashes contributes significantly to the burden of disease in many countries. In South Africa, research into the relationship between alcohol intoxication and other driver risk behaviours is limited and variable, constraining appropriate and effective policy and programmatic options and interventions.

Objectives. To examine the risk for fatal crashes attributed to driver alcohol intoxication relative to speeding and other driver risk behaviours across a range of key crash and vehicle characteristics and temporal variables.

Methods. The study used a sample of fatal crashes drawn from the Road Traffic Management Corporation database for the period 2016 2018, comprising 13074 fatal crashes. An overview of the sample data is provided using descriptive statistics. Following this, logistic regression modelling was undertaken to examine and clarify the risk for alcohol-attributed fatal crashes against that for speeding and a combined category of all other driver risks using variables relating to crash complexity, vehicle characteristics and regulation, and temporal variables for day/night, weekday/weekend and vacation/non-vacation periods.

Results. Compared with fatal crashes involving only the driver, the study revealed a significantly greater risk for alcohol-attributed fatal crashes in instances involving more complexity as measured by involvement of other road users (pedestrians and other drivers). Additionally, the risk for alcohol-attributed fatal crashes was significantly greater for light vehicles and buses/midibuses compared with trucks. Road users were also at greater risk for such crashes at night, over long and regular weekends, and during non-vacation periods of the year.

Conclusions. Improved enforcement to prevent alcohol intoxication using roadblocks is required in a focused manner during specific temporal periods (at night, over weekends and during non-vacation periods), while enforcement to prevent speeding and other driver risks should be prioritised during other periods using speed monitoring and mobile visible policing, respectively. There is an urgent need to improve the current measurement of alcohol attribution in fatal crashes, to ensure more accurate estimation of prevalence, and to improve analysis and understanding of the compound impact of alcohol intoxication on all other driver risk behaviours and associated fatal crashes.
\end{abstract}

S Afr Med J 2021;111(10):968-973. https://doi.org/10.7196/SAMJ.2021.v111i10.15057

\section{Burden of alcohol-related crashes}

Globally, alcohol is implicated as a significant contributor to the enormous burden of injury-related mortality and morbidity from road traffic crashes, including associated psychosocial and mental health consequences of such crashes. ${ }^{[1]}$ In Australia, 30\% of all fatally injured drivers had blood alcohol concentrations (BACs) at or above the legal limit of $0.05 \mathrm{~g} / 100 \mathrm{~mL}$; in Canada $38.3 \%$ of all fatally injured drivers had BACs $>0.08 \mathrm{~g} / 100 \mathrm{~mL}$; in China $34 \%$ of all fatal road crash victims had BACs between 0.02 and $0.08 \mathrm{~g} / 100 \mathrm{~mL} ;{ }^{[2]}$ in Europe 25\% of all road fatalities had BACs above the various country limits; and in the USA $29 \%$ of all traffic fatalities had BACs $\geq 0.08 \mathrm{~g} / 100 \mathrm{~mL} \cdot{ }^{[3]} \mathrm{In}$ South African (SA) drivers, a review of various studies that included selected driver samples in various geographical and clinical settings indicates prevalence estimates of driver intoxication between 33\% and $69 \% \cdot{ }^{[4]}$

Beyond drivers, the impact of alcohol intoxication on all road users in SA has been reported by the South African Road Traffic Management Corporation (RTMC) and other research as far-reaching and profound for families, communities and the economy. ${ }^{[5,6]}$ In 2018 , the cumulative cost for all road crashes in the country was estimated at ZAR166 billion, with alcohol considered a significant contributor to the total burden of crashes. However, despite the estimated likely impact of alcohol in road fatalities, there is somewhat limited and variable SA research on the extent of alcohol's contribution to the country's fatal crash burden. This lack has a constraining impact on developing and implementing the policy, prevention and control programming deemed necessary to mitigate such crashes and their consequences for individuals, families and society.

\section{Alcohol intoxication and driver risk behaviours}

Behavioural risk factors are the most commonly reported risks associated with road traffic crashes and injuries, ${ }^{[7]}$ with alcoholimpaired driving a leading behavioural risk factor, along with others relating to excessive and inappropriate driving speeds, inadequate or inappropriate use of safety restraints and devices, driver distraction, and drug-impaired driving. ${ }^{[8]}$ In SA, human behavioural risk factors are estimated to contribute to $89 \%$ of road traffic crashes. ${ }^{[9]}$

Psychologically, drinking and driving has been associated with sensation-seeking, and it is also a predictor of other risky driving behaviours, such as speeding, unsafe lane changes and passing other vehicles, tailgating or short following distances, and failure to 
slow down or stop when appropriate. ${ }^{[10]}$ The use of alcohol is also associated with more risk-prone changes in safety attitudes, such as to seat belt use, helmet use and speed choice. ${ }^{[11,12]}$ Importantly, alcohol has been shown to impair driving performance even in quantities less than country-specified legal limits, with growing evidence indicating that there is no BAC threshold below which some degree of impairment does not occur. ${ }^{[13]}$

In SA, enforcement to prevent high-risk driving behaviours, along with related education and awareness-raising campaigns, forms the major thrust in the national response to the burden of crashes and associated fatalities. Traffic law enforcement performance and infringement data collected in all provinces over a 22 -month period from April 2013 to January 2015 $5^{[14]}$ indicate that 1034199 vehicles were stopped for routine inspections and 212588 drivers were tested for alcohol. The enforcement operations resulted in a monthly average of 8196 arrests, of which 2742 were for 'drunken driving. In addition, a total monthly average of 308444 moving offences was reported, including 148688 from speed-related operations.

The above enforcement actions to prevent diverse driver infringements have to a large extent been hampered by a lack of scientific evidence that permits better prioritisation of resources and efforts. Additionally, as highlighted by national safety experts responsible for submitting country data to the World Health Organization, there is considerable scope for improvement in the enforcement of driver behaviour in the country. ${ }^{[15]}$

\section{Characteristics of alcohol-related crashes}

Several key characteristics of road traffic crashes have been identified in alcohol-related crashes. Research has highlighted the influence of a range of effects pertaining to the nature and circumstances of crashes, including type of crash and type of vehicle. ${ }^{[16]}$ There have also been descriptions of variations in road traffic fatalities at the national level, ${ }^{[17,18]}$ and analysis demonstrating alcohol to be a significant predictor in weekly variation of road traffic fatalities when controlling for other temporospatial effects such as traffic exposure, weather conditions and types of holiday ${ }^{[19]}$ Importantly, such crash characteristics have not been analysed in the context of and relative to different driver behaviours, this being critical to well-informed prioritisation of resources for different types of driver behaviourbased interventions.

\section{Objectives}

To examine the extent to which driver alcohol intoxication, relative to risk behaviours such as speeding and all other driver risks, places road users in SA at increased risk of being involved in fatal road traffic crashes. The role of driver alcohol intoxication is examined in the context of key outcome factors such as type of road user fatality, and influence factors such as type of crash, vehicle type, and various temporal factors relating to time of day and week and vacation period.

\section{Methods}

\section{Primary data collection}

The data were obtained from the RTMC administrative national database of fatal road traffic crashes. As part of its legislative statutory mandate, the RTMC routinely collects and maintains data on all road traffic crashes in the country, including those for which there are one or more fatalities (designated as fatal crashes). The data are collected by the RTMC in partnership with the South African Police Service (SAPS), using the Culpable Homicide Observation Form (ChoCOR) (available as a supplementary file at http://samj.org.za/public/sup/15057.pdf). ${ }^{[9]}$
Using SAPS daily reports, the RTMC captures, processes and verifies all data that are compiled into its national database. ${ }^{[9]}$ The database includes information on key features of the crash, the vehicles involved, and the characteristics of victims where injuries are involved. All data supplied by the RTMC were completely anonymised prior to delivery to the research team, with no information present or available in the dataset to identify individual crashes or crash victims.

\section{Sample}

Based on consultations with the RTMC, the sample for this study was restricted to all fatal crashes in which driver error was specifically attributed as the primary cause for the fatal crash. This specification excluded fatal crashes for which other factors were attributed as the primary cause for the fatal crash, such as road, weather and vehicle factors, and factors relating to other road users such as pedestrians. A further specification to ensure data completeness was to restrict the sample to fatal crashes that occurred during the period 1 January 2016 - 31 December 2018. This selected sample comprised 13074 fatal crashes, involving 19748 vehicles and resulting in 17706 road user fatalities (passenger, pedestrian and driver).

\section{Analysis}

Descriptive statistics and logistic regression analysis were used to examine key sample characteristics and risk for fatality across a series of control and key explanatory factors. Analysis was performed using the Statistical Package for Social Sciences (SPSS) version 25 (IBM Corp., USA), with a $p<0.05$ significance level.

\section{Variables}

\section{Outcome variable}

The primary analysis variable was the risk for a fatal crash in driver alcohol-attributed fatal crashes relative to fatal crashes attributed to either speeding or all other driver risk behaviours. The attribution of fatal crashes to driver alcohol intoxication was made by the police officer investigating the crash. This determination derives from the inputs received by the crash site investigator from designated first responders (police/paramedics) at the crash site. The determination of driver intoxication by designated first responders is based on established standardised operating protocols for such determination in traffic crashes as detailed in applicable SAPS internal policies and training manuals (Col. Thumba Govender, SAPS, personal communication 4 November 2020 and 15 March 2021).

Crash attribution. To align the driver risk behaviours to identified established law enforcement and intervention strategies, the various risk behaviours were recoded into the following categories, with associated interventions indicated in parentheses:

- Driver intoxication (breathalyser tests at roadblocks)

- Driver speeding (active and passive speed monitoring)

- All other driver risk behaviours, comprising a combination of the following behaviours: turn/U-turn, overtaking, following too closely, fatigue/fell asleep, disregard for traffic lights/signs, and cellphone distraction (visible mobile policing).

\section{Explanatory variables}

Crash type. The original RTMC data comprise multiple crash types. To properly account for the degree of complexity in the contextual environment within which the fatal crash occurred, and the associated difficulty in managing this complexity by the driver, three crash type categories were formulated:

- Driver only. This crash excludes other road users and the risk behaviour relates only to that of drivers themselves, such as a single vehicle that overturns, leaves the road or collides with a fixed 
object. This is a low-complexity context and was set as the reference category in the logistic regression analysis.

- Driver-pedestrian. This context has a higher level of complexity, as the driver has to negotiate the use of the road with another person, specifically a pedestrian. However, the pedestrian is moving at a slower speed than the driver, so this is a moderate-complexity context.

- Driver-driver. This third type of context is the most complex, as the driver is negotiating use of the road with other drivers, all travelling at similar or varying speeds. This is the high-complexity context.

Vehicle type. The vehicle type reflects the primary vehicle per fatal crash, i.e. the vehicle most likely to be associated with the cause of the fatal crash, as determined by the investigating officer. The various vehicle types were recoded to reflect the increasing degree of regulation of drivers and their vehicles, as follows:

- Light vehicle - all private cars and light delivery vehicles (least regulated)

- Public transport - only minibus taxis

- Public transport - midibuses and buses

- Goods transport - trucks and all commercial vehicles with a gross vehicle mass exceeding $3500 \mathrm{~kg}$ (the most regulated sector) (reference category).

Day period. This variable reflects the time of day in a 24-hour period, differentiated into night (19h00 - 06h00) and day (07h00 - 18h00) (reference category).

Vacation period. This variable covers all school vacation periods for the review period, and includes public holidays, differentiated as non-vacation and vacation (reference category).

Week period. This variable differentiates the weekday from regular and long weekends, with all weekends beginning at $16 \mathrm{~h} 00$ on the day preceding the start of the weekend period and ending at $05 \mathrm{~h} 00$ on the day following the weekend period. The categories were long weekend, regular weekend and weekday (reference category).

In addition, spatial effects were also considered in the descriptive analysis through inclusion of the following two variables:

Province of crash. Location of the crash in relation to the country's nine provinces.

Municipality of crash. Providing an indication of urbanity through demarcation of the municipality as being either metropolitan or local, based on SA census categorisations.

\section{Results}

\section{Descriptive analysis}

In terms of driver risk behaviours, the majority of crashes were attributed to speeding (52.3\%), followed by all other driver risks $(42.2 \%)$ and then driver alcohol intoxication (5.5\%). Three-quarters (74.7\%) of crashes resulted in a single fatality, $12.5 \%$ resulted in two fatalities and $7.5 \%$ resulted in three or more fatalities. Most victims of fatal crashes were passengers $(48.7 \%)$, followed by drivers $(40.7 \%)$ and then pedestrians (10.6\%), and more than three-quarters were male $(76.8 \%)$. Four out of five fatal crashes involved a light vehicle while $11.9 \%$ involved various types of buses. Compared with other times, slightly more fatal crashes occurred during non-vacation periods (69.5\%), at night (55.6\%), and over regular weekends (51.8\%). Further clarification of the descriptive statistics, including spatial differentiation, is provided in Table 1.

\section{Logistic regression analysis}

Multinomial logistic regression modelling was performed examining the risks associated with alcohol compared with speeding and all other driver risk behaviours in the context of crash type, vehicle type and temporal characteristics (Table 2).

The logistic regression modelling revealed non-significant differences in the relative risk of fatalities for drivers, pedestrians and passengers in alcohol-attributed crashes compared with crashes attributed to speeding and all other driver risks, with two notable exceptions: the fatality risk for passengers was marginally higher for alcohol compared with speeding (odds ratio (OR) 1.093; 95\% confidence interval (CI) 1.001 - 1.192), and the risk for driver fatality was lower for alcohol compared with all other driver risk behaviours (OR 0.811; 95\% CI 0.660 - 0.996).

Relative to speeding, alcohol was found to be a greater risk factor for fatal crashes involving multiple drivers compared with a single driver (OR 3.255; 95\% CI 2.623 - 4.039) and a substantially greater risk factor for fatal crashes involving drivers and pedestrians rather than drivers alone (OR 7.760; 95\% CI 4.899 - 12.291). Relative to all other driver risks, alcohol-related crashes carried a much lower risk

\begin{tabular}{|c|c|c|}
\hline & $\begin{array}{l}\text { Total fatalities } \\
(N=17 \text { 706) }, n(\%)\end{array}$ & $\begin{array}{l}\text { Total crashes } \\
(N=13074), n(\%)\end{array}$ \\
\hline \multicolumn{3}{|l|}{ Driver behaviour } \\
\hline Alcohol & $949(5.4)$ & $725(5.5)$ \\
\hline Speeding & $8666(48.9)$ & $6838(52.3)$ \\
\hline Other driver risks & $8091(45.7)$ & $5511(42.2)$ \\
\hline \multicolumn{3}{|l|}{ Crash type* } \\
\hline Driver-driver & $7548(42.6)$ & $4752(36.3)$ \\
\hline Driver-pedestrian & $1383(7.8)$ & $1312(10.0)$ \\
\hline Driver only & $6981(39.4)$ & $5443(41.6)$ \\
\hline \multicolumn{3}{|l|}{ Vehicle type $^{*}$} \\
\hline Light vehicle & $13210(74.6)$ & $9880(75.6)$ \\
\hline Minibus & $1578(8.9)$ & $987(7.5)$ \\
\hline Bus and midibus & $418(2.4)$ & $203(1.6)$ \\
\hline Truck & $1533(8.7)$ & $1100(8.4)$ \\
\hline \multicolumn{3}{|l|}{ Day period } \\
\hline Night & $9844(55.6)$ & $7195(55.0)$ \\
\hline Day & $7862(44.4)$ & $5879(45.0)$ \\
\hline \multicolumn{3}{|l|}{ Week period } \\
\hline Long weekend & $2371(13.4)$ & $1659(12.7)$ \\
\hline Regular weekend & $9169(51.8)$ & $6673(51.0)$ \\
\hline Weekday & $6166(34.8)$ & $4742(36.3)$ \\
\hline \multicolumn{3}{|l|}{ Vacation period } \\
\hline Non-vacation & $12307(69.5)$ & $9183(70.2)$ \\
\hline Vacation & $5399(30.5)$ & $3891(29.8)$ \\
\hline \multicolumn{3}{|l|}{ Municipality type } \\
\hline Local & $13195(74.5)$ & $9408(72.0)$ \\
\hline Metro & $4511(25.5)$ & $3666(28.0)$ \\
\hline \multicolumn{3}{|l|}{ Province } \\
\hline Eastern Cape & $1953(11.0)$ & $1405(10.7)$ \\
\hline Free State & $1309(7.4)$ & $918(7.0)$ \\
\hline Gauteng & $3302(18.6)$ & $2670(20.4)$ \\
\hline KwaZulu-Natal & $2842(16.1)$ & $2120(16.2)$ \\
\hline Limpopo & $2439(13.8)$ & $1708(13.1)$ \\
\hline Mpumalanga & $2245(12.7)$ & $1578(12.1)$ \\
\hline Northern Cape & $612(3.5)$ & $441(3.4)$ \\
\hline North West & $1460(8.2)$ & $1055(8.1)$ \\
\hline Western Cape & $1544(8.7)$ & $1179(9.0)$ \\
\hline
\end{tabular}

*Totals do not add to $100 \%$ owing to missing values for some categories of the variable. 
Table 2. Logistic regression modelling of driver intoxication relative to other driver risk behaviours

\begin{tabular}{|c|c|c|c|c|}
\hline & \multicolumn{2}{|c|}{ Alcohol compared with speeding } & \multicolumn{2}{|c|}{ Alcohol compared with other driver risks } \\
\hline & OR & $95 \%$ CI & OR & $95 \%$ CI \\
\hline \multicolumn{5}{|l|}{ Road user fatalities } \\
\hline Driver & 0.876 & $0.714-1.076$ & $0.811^{*}$ & $0.660-0.996$ \\
\hline Passenger & $1.093^{*}$ & $1.001-1.192$ & 1.034 & $0.948-1.128$ \\
\hline Pedestrian & 1.228 & $0.841-1.794$ & 1.452 & $0.994-2.120$ \\
\hline \multicolumn{5}{|l|}{ Crash type } \\
\hline Driver-driver & $3.255^{*}$ & $2.623-4.039$ & $0.128^{*}$ & $0.103-0.159$ \\
\hline Driver-pedestrian & $7.760^{*}$ & $4.899-12.291$ & 0.669 & $0.421-1.061$ \\
\hline Driver only (reference) & - & & - & \\
\hline \multicolumn{5}{|l|}{ Vehicle type } \\
\hline Light vehicle & $1.584^{*}$ & $1.051-2.386$ & $1.631^{*}$ & $1.088-2.444$ \\
\hline Minibus & 1.351 & $0.815-2.242$ & 1.246 & $0.755-2.057$ \\
\hline Bus and midibus & $2.536^{*}$ & $1.232-5.220$ & 1.800 & $0.895-3.617$ \\
\hline Truck (reference) & - & & - & \\
\hline \multicolumn{5}{|l|}{ Day period } \\
\hline Night & $1.435^{*}$ & $1.195-1.724$ & $1.377^{\star}$ & $1.143-1.659$ \\
\hline Day (reference) & - & & - & \\
\hline \multicolumn{5}{|l|}{ Week period } \\
\hline Long weekend & $1.986^{*}$ & $1.466-2.691$ & $2.177^{\star}$ & $1.599-2.965$ \\
\hline Weekday (reference) & - & & - & \\
\hline \multicolumn{5}{|l|}{ Vacation period } \\
\hline Non-vacation & $1.351^{*}$ & $1.103-1.656$ & $1.352^{*}$ & $1.100-1.661$ \\
\hline Vacation (reference) & - & & - & \\
\hline
\end{tabular}

for fatal crashes with multiple drivers compared with those involving single drivers (OR 0.128; 95\% CI 0.103 - 0.159).

The type of vehicle was also important in defined instances. Relative to trucks, light vehicles and buses/midibuses were significantly more likely to be involved in fatal crashes attributed to alcohol rather than speeding (OR 1.584; 95\% CI $1.051-2.386$ and OR 2.536; 95\% CI 1.232 - 5.220, respectively). Similarly, relative to trucks, light vehicles were significantly more likely to be involved in fatal crashes attributed to alcohol rather than any other driver risk behaviour (OR 1.631; 95\% CI 1.088 - 2.444).

Analysis of temporal characteristics revealed several significant differences. Firstly, relative to both speeding and other driver risks, alcohol was found to be a greater risk factor for fatal crashes occurring at night rather than during the day (OR 1.435; 95\% CI $1.195-1.724$ and OR 1.377; 95\% CI 1.143 - 1.659, respectively). Relative to both speeding and other driver risks, alcohol was also shown to carry twice the risk for fatal crashes occurring over regular weekends compared with weekdays (OR 1.917; 95\% CI 1.546 - 2.378 and OR 2.055; 95\% CI $1.653-2.554$, respectively) and for crashes occurring over long weekends compared with weekdays (OR 1.986; 95\% CI 1.466 - 2.691 and OR 2.177; 95\% CI $1.599-2.965$, respectively). Finally, relative to both speeding and other driver risks, alcohol was also shown to be a greater risk factor for fatal crashes occurring during non-vacation periods compared with vacation periods (OR 1.351; 95\% CI 1.103 1.656 and OR 1.352; 95\% CI 1.100 - 1.661, respectively).

\section{Discussion}

Alcohol was found to be a greater risk factor for crashes involving other road users than for crashes with single road users (drivers). This finding bears on the dynamic and compound nature of the road traffic environment, which requires motorists to continuously process and manage complexity for optimal decision-making and response. Driver alcohol intoxication, with its direct negative impacts on critical psychomotor and cognitive skills, significantly diminishes the overall capacity to anticipate and respond to potential and actual hazardous situations. ${ }^{[11,12]}$

The substantially higher risk found for pedestrians in fatal crashes may relate to the complexity inherent in drivers and pedestrians needing to negotiate use of common road space but at significantly different speeds, along with the greater injury severity for pedestrians from such crashes. Additionally, it may reflect the added complexity for drivers to negotiate the unpredictability of pedestrian behaviour, especially in suboptimal settings such as those with inadequate pedestrian infrastructure for walking and crossing and conditions where lighting is poor. ${ }^{[20,21]}$

The elevated risk to pedestrians is also likely to be due to a general lack of safe pedestrian-related infrastructure, ${ }^{[20]}$ as well as their engagement in activities in the urban environment that result in increased exposure to pedestrian-vehicle conflict. One such behaviour is alcohol intoxication among vulnerable road users, with evidence showing a positive association between the availability of alcohol outlets and crash injury risk for both pedestrians and cyclists. ${ }^{[21]}$

Alcohol was found to pose an increased risk for crashes involving both light vehicles and buses relative to trucks. The significantly diminished risk for trucks may reflect the greater regulation and enforcement of such vehicles on the road. It is notable that this effect was not evident for crashes involving minibus taxis, consistent with some studies pointing to a high prevalence of other illicit substance use among taxi drivers, including the use of drugs such as 'tik', 'nyaope' and 'wonga. ${ }^{[22,23]}$ These illicit 'cocktails' contain various formulations of methamphetamine, cannabis, heroin and other substances and are likely to be used for the purposes of enhancing driver and operational performance, specifically in a manner that increases frequency of trips and boosts passenger loads in order 
to meet and exceed income-linked daily targets. ${ }^{[24]}$ The increased alcohol risk in fatal crashes involving buses is especially concerning for two important reasons: (i) these are bulk passenger vehicles where the risk of loss of multiple lives is high; and (ii) as they are public permit carriers, it is expected that such vehicles would be subject to similar or even greater regulation and enforcement protocols than trucks conveying cargo goods.

The higher alcohol-related risk for fatal crashes during night time and all weekend periods is consistent with several previous fatal crash and injury analyses undertaken in SA. ${ }^{[17-19]}$ Night driving is generally associated with increased risk owing to diminished visibility and greater exhaustion, while weekends tend to be associated with higher risk owing to relatively greater travel-related exposure from leisure-related activities, including driving while under the influence of alcohol. In terms of vacation periods, alcohol presents a greater risk for fatal crashes during non-vacation periods. This may reflect the fact that while vacation periods are associated with increased leisure activities and consumption of alcohol, this risk may be offset with decreased travel exposure resulting from alcohol consumption occurring principally within the residential context. It may also reflect the greater frequency and visibility of campaign-related interventions during such vacation periods.

\section{Recommendations}

Several key recommendations derive from this research.

Firstly, in terms of specific programmatic interventions by traffic management authorities, it is necessary to assign greater priority to targeting driver intoxication during the night, over weekends (both regular and long weekends), and across all the non-vacation periods of the year. This can be achieved mainly through roadblocks. Relatively greater priority should be assigned to the targeting of nonalcohol-related driver risk behaviours during other periods (daytime, weekday and vacation periods), this being achieved by enforcement methods such as speed monitoring for speeding and mobile visible policing for all other driver risk behaviours.

Secondly, it is essential to review and improve the measurement of alcohol in fatal crash data. The current estimate of alcohol-related fatal crashes by the RTMC is by all accounts a significant underestimate, given other research from both the local and global contexts. The estimate derives from the current RTMC measurement methodology, which records driver intoxication as a category that is mutually exclusive of all other driver risk behaviour categories, meaning that alcohol intoxication can only be measured when all other driver risk behaviours are excluded. This methodology therefore discounts the fact that alcohol intoxication co-occurs and exacerbates the risks associated with other driver risks such as speeding, disregarding traffic lights and signs, overtaking, fatigue, falling asleep, and cell phone (mobile phone) distraction. Accordingly, it is necessary to record driver intoxication in fatal crash data as a category of risk that overlaps other categories of driver risk behaviours, rather than as a mutually exclusive category. In this manner, the role of alcohol intoxication will be manifest regardless of any other driver risk behaviour, thereby enabling proper analysis of the compounding effect of alcohol intoxication on all driver risk behaviours.

Thirdly, and accounting for the proposed overlapping measurement of alcohol intoxication with other driver risk behaviours, this research highlighted that speeding accounts for just over half of all driver risks while overtaking, which is closely linked with relatively higher driver speeds, which account for one-quarter. In sum, three-quarters of fatal crashes are related to travelling at excessive or inappropriate speed. There is an urgent need for more research into these specific driver risk behaviours, including the interaction with alcohol intoxication, to build more robust programmatic interventions directed at behavioural change among motorists and more effective detection and enforcement by traffic management authorities.

\section{Study limitations}

The results of this study are circumscribed by two notable limitations. Firstly, and most importantly, there are important shortcomings in the current measurement of alcohol intoxication as the attribution for fatal crashes, resulting in an arguably gross underestimate of its likely prevalence and impact on both crashes and fatalities. Secondly, the attribution of a specific driver risk behaviour as the likely cause of a fatal crash is based on the judgement of the crash investigator in a post-hoc manner, and the possibility exists that it may deviate from the final attribution in some instances, particularly when there is insufficient information in the accident investigation.

\section{Conclusions}

There is a need for prioritisation of driver intoxication monitoring and detection at night and over regular and long weekends, throughout the non-vacation periods of the year. This can be successfully achieved through roadblocks. Other driver behaviour infringements, particularly speeding, should receive greater priority during other temporal periods (daytime, weekdays and vacation periods), using other enforcement methods. There is a pressing need for review and revision of the measurement of driver intoxication in fatal crashes. The current measurement assumes that the different categories of driver risk behaviours are mutually exclusive. Revisions are necessary to ensure measurement of risk as overlapping categories, to better understand the exacerbating impact of alcohol intoxication in the context of other driver behaviours (such as speeding, following too closely, disregarding traffic signals, etc.). Finally, in terms of other road users, there is a need for greater focus on risks for fatality among pedestrians.

\section{Declaration. None}

Acknowledgements. The research reported in this publication was supported by the South African Medical Research Council, the University of South Africa and the RTMC. The content and findings reported are the sole deductions, views and responsibility of the researchers and do not reflect the official positions and sentiments of the South African Medical Research Council, the University of South Africa or the RTMC.

Author contributions. RG, AvN and AS conceptualised the study. DR oversaw data collation and RG refined and cleaned the final dataset. RG with AS led the data analysis, with all authors involved in the interpretation of findings and write-up of the manuscript. All authors have read and approved the manuscript.

Funding. This research was funded by the RTMC.

Conflicts of interest. None.

1. World Health Organization. Road traffic injuries. 2019. https://www.who.int/news-room/fact-sheets detail/road-traffic-injuries (accessed 10 May 2020).

2. Chen $\mathrm{H}, \mathrm{Chen} \mathrm{Q}$, Chen L, Zhang G. Analysis of risk factors affecting driver injury and crash injury with drivers under the influence of alcohol (DUI) and non-DUI. Traffic Inj Prev 2016;17(8):796-802. with drivers under the influence of alcohol (DUI)

3. National Highway Traffic Safety Administration. Traffic safety facts: Alcohol impaired driving Washington, DC: NHTSA, 2018. https://crashstats.nhtsa.dot.gov/api/public/viewpublication/812864 (accessed 20 May 2020)

4. Govender R, Sukhai A, van Niekerk A. Driver intoxication and fatal crashes in South Africa: Alcohol as a risk factor for fatal crashes and fatalities, 2016 to 2018. Road Traffic Management Corporation, South Africa, 2020. http://www.rtmc.co.za/images/rtmc/docs/research_dev_rep/Driver\%20intoxication\%20 and\%20fatal\%20crashes\%20Report\%20-\%20March_2020.pdf (accessed 20 May 2020).

5. Simons A, Marais L, Hornsby N, Swart L, Seedat M, van Niekerk A. Alcohol and its implications for road traffic crashes in South Africa. Road Traffic Management Corporation, South Africa, 2019. http:// www.rtmc.co.za/images/rtmc/docs/research_dev_rep/Alcohol\%20and\%20its\%20implications $\% 20$ for\%20RTCs\%20in\%20South\%20Africa\%20Phase $\% 20 \mathrm{~A} \% 20 \mathrm{March} \% 202019$.pdf (accessed $15 \mathrm{April}$ 2020). 
6. Labuschagne FJJ. Cost of crashes in South Africa. Pretoria: Road Traffic Management Corporation and Council for Scientific and Industrial Research, 2016. http://www.satc.org.za/archive17/assets/2c labuschagne.pdf (accessed 15 April 2020).

7. Razzaghi A, Soori H, Kavousi A, Abadi A, Khosravi A, Alipour A. Risk factors of deaths related to Razzaghi A, Soori H, Kavousi A, Abadi A, Khosravi A, Alipour A. Risk factors of deaths related to
road traffic crashes in World Health Organization regions: A systematic review. Arch Trauma Res 2019;8(2):57-86. https://doi.org/10.4103/atratr_59_19

8. World Health Organization. Global status report on road safety 2018. Geneva: WHO, 2018. https:// www.who.int/publications//ititem/global-status-report-on-road-safety-2018 (accessed 10 May 2020). 9. Road Traffic Management Corporation, South Africa. State of road safety report. 2018. https://www. 15 April 2020).

10. Shyhalla, K. Alcohol involvement and other risky driver behaviors: Effects on crash initiation and crash severity. Traffic Inj Prev 2014;15(4):325-334. https://doi.org/10.1080/15389588.2013.822491

11. Heng K, Hargarten S, Layde P, Craven A, Zhu S. Moderate alcohol intake and motor vehicle crashes: The conflict between health advantage and at-risk use. Alcohol Alcohol 2006;41(4):451-454. https:// doi.org/10.1093/alcalc/agh258

12. World Health Organization. Global status report on alcohol and health. Geneva: WHO, 2014 https://apps. who.int/iris/bitstream/handle/10665/112736/9789240692763 eng pdf?sequence= (accessed 10 May 2020)

13. Ogden EJD, Moskowitz H. Effects of alcohol and other drugs on driver performance. Traffic Inj Prev 2004:5(3):185-198. https://doi.org/10.1080/15389580490465201

14. Road Traffic Management Corporation, South Africa. Traffic offence survey. Pretoria: RTMC, 2015 Road Traffic Management Corporation, South Africa. Traffic offence survey. Pretoria: RTMC, 2015. https://www.rtme.co.za/images/rtmc/docs/research_dev_rep/31\%20Mar.

15. Sukhai A, Jones AP. Urban density, deprivation and road safety: A small area study in the eThekwini Sukhai A, Jones AP. Urban density, deprivation and road safety: A small area study in
Metropolitan Area, South Africa. Afr Saf Promot J Inj Violence Prev 2014;12(2):10-29.

16. Road Traffic Management Corporation. State of road safety report: Calendar, January - Decembe 2018. RTMC and National Department of Transport, South Africa, 2019. http://www.rtmc.co.za/ images/rtmc/docs/traffic_reports/calendar/calendar_jan_dec_2018.pdf (accessed 15 April 2020).
17. Sukhai A, Jones AP, Haynes R. Epidemiology and risk of road traffic mortality in South Africa. S Afr Geogr J 2009;91(1):4-15. https://doi.org/10.1080/03736245.2009.9725325

18. Sukhai A, Jones AP, Love BS, Haynes R. Temporal variations in road traffic fatalities in South Africa. Accid Anal Prev 2011;43(1):421-428. https://doi.org/10.1016/j.aap.2010.09.012

19. Baker PC. Collision course: Why are cars killing more and more pedestrians? Guardian, 3 October 2019. https://www.theguardian.com/technology/2019/oct/03/collision-course-pedestrian-deathssing-driverless-cars (accessed 25 May 2020).

20. World Health Organization, FIA Foundation for the Automobile and Society, Global Road Safety Partnership and World Bank. Pedestrian safety: A road safety manual for decisionmakers and practitioners. Geneva: WHO, 2013. https://apps.who.int/iris/bitstream/ handle/10665/79753/9789241505352_eng.pdf;jsessionid=8FA410EBE756FFB8DCC8855C7231C361? sequence $=1$ (accessed 15 April 2020).

21. DiMaggio C, Mooney S, Frangos S, Wall S. Spatial analysis of the association of alcohol outlets and alcohol-related pedestrian/bicyclist injuries in New York City. Inj Epidemiol 2016;3(1):11. https://doi. org/10.1186/s40621-016-0076-5

22. Moodley L. An investigative study into the knowledge and perceptions of illicit drug trafficking into and within Durban, KwaZulu-Natal, South Africa. University of KwaZulu-Natal, South Africa, 2016. https://researchspace.ukzn.ac.za/handle/10413/14833 (accessed 20 May 2020).

23. Randall L. Is profit taking precedence over passengers' safety? Focus on Transport and Logistics, Feature Issue 4, 2018. http://www.focusontransport.co.za/is-profit-taking-precedence-overpassengers-safety/ (accessed 2 June 2020)

24. Arrive Alive Minibus taxis and road safety. 2020. https://wwwarrivealive mobi/minibus-taxis-androad-safety (accessed 2 June 2020).

Accepted 25 May 2021. 